\section{Psychiatrische Komorbidität und Suchtbehandlung}

Zusammenfassung: Der vorliegende, den Themenschwerpunkt „Komorbidität“ einleitende Beitrag liefert einen Überblick zur Entwicklung der Komorbiditätsforschung und die in diesem Gebiet relevanten Fragestellungen. Ferner wird auf theoretische Zusammenhänge verwiesen, und es werden Konsequenzen für weitere Forschungsaktivitäten diskutiert.

Schlüsselwörter: Komorbidität - Substanzabhängigkeit Diagnostik - Interventionsforschung

Psychiatric Comorbidity and Treatment of Addiction: The following article is an introduction of the special topic section “comorbidity” of the new scientific journal „Suchttherapie" ("Addiction Therapy"). It provides an overview of the development and relevant issues of comorbidity research, and the theoretical implications and consequences of research are discussed.

Key Words: Comorbidity - Substance Dependence Diagnosis - Treatment Evaluation

Die Diskussion um Komorbidität im engeren Sinne reicht zurück bis in die frühen 80er Jahre. Die Relevanz von Verhaltensauffälligkeiten und psychiatrischen Problemen bei Heroinabhängigen etwa in Methadonprogrammen wurden damals von Woody et al. [1] und anderen Kollegen der Arbeitsgruppe in Philadelphia [2] herausgearbeitet. In der europäischen Diskussion rückte Komorbidität erst in den 90er Jahren stärker in den Mittelpunkt. Sie ist gewissermaßen ein wissenschaftliches Schwerpunktthema der 90er Jahre - vergleichbar anderen Themen insbesondere im Drogenfeld wie „Niedrigschwelligkeit“, „Substitution“ oder „Schadensbegrenzung“.

Trotz relativ wenig eigenständiger klinischer Forschung in dem Bereich der Komorbidität reagierte die Praxis in verschiedenen Teilen der Bundesrepublik mit der Entwicklung von Pilotprojekten oder spezialisierten Einzelangeboten auf die klinische Herausforderung der Koinzidenz von schweren psychischen Erkrankungen und Sucht [3]. Einige der im Rahmen des Heftschwerpunktes vorgestellten Einrichtungen entstanden zu dieser Zeit und können mittlerweile über eigene, überwiegend positive Erfahrungen berichten.

Suchttherapie 2000; $1: 3-7$

(c) Georg Thieme Verlag Stuttgart · New York

ISSN 1439-9903
Michael Krausz, Peter Degkwitz, Uwe Verthein

Zentrum für Interdisziplinäre Suchtforschung der

Universität Hamburg (ZIS)
Die verstärkte klinische Aufmerksamkeit für dieses Phänomen geht einher mit einer Veränderung der Klassifikation von ICD-9 zu ICD-10 [4] oder den entsprechenden amerikanischen Diagnostik-Systemen. In Abweichung zu der hierarchischen Diagnostik wurde das Komorbiditätsprinzip als wesentliches Element deskriptiver Psychopathologie eingeführt, mit dem Grundgedanken, alle behandlungsbedürftigen psychischen Störungen ohne kausale Verknüpfung oder Erkrankungshierarchien zu beschreiben und damit eine bessere Grundlage für die Verlaufsdokumentation und die Therapieplanung zu liefern.

Dass dies gerade in der ätiologischen Debatte sowie der Interpretation süchtigen Verhaltens noch keine Problemlösung darstellt, sondern lediglich bessere Instrumente zur Deskription bereitstellt, zeigt das Beispiel der Koinzidenz von Persönlichkeitsstörungen (F6-Störungen nach ICD-10) und Störungen durch den Konsum psychotroper Substanzen (F1Störungen). In der wissenschaftlichen Literatur wurden durchschnittliche Prävalenzraten zwischen 40 und 80\% in Abhängigkeit zum verwendeten diagnostischen Verfahren sowie der konsumierten Substanz genannt [5], ohne dass dies für die Entwicklung entsprechender Therapiekonzepte Konsequenzen gehabt hätte [6]. Dies hängt allerdings auch mit dem insgesamt vagen Konstrukt der Persönlichkeitsstörung und der Operationalisierung verschiedener Syndrombereiche zusammen. So gibt es zwischen verschiedenen Leitsymptomen, z.B. der antisozialen, paranoiden oder Borderline-Persönlichkeitsstörung und typischen Verhaltensweisen von Suchtpatienten, die vielfach durch die Illegalität der Substanzen bedingt sind, starke Überschneidungen, die eine entsprechende diagnostische Einschätzung erschweren [7]. Damit kann die Diagnose der Persönlichkeitsstörung häufig als nachträglicher Grund für institutionellen Misserfolg herhalten.

Vor dem Hintergrund dieser Erfahrung ist es sinnvoll, die Komorbiditätsforschung immer wieder unter den Anspruch der praktischen Konsequenzen zu stellen. Sicher sind von den erforderlichen epidemiologischen Arbeiten nicht unmittelbare Konsequenzen ableitbar; aber nach langjähriger Arbeit muss die Diskussion über diesen Aspekt hinausgehen, wenn sie Defizite nicht nur feststellen, sondern zu ihrer Auflösung beitragen will.

Begonnen hat die Forschung mit den empirisch gefundenen Zusammenhängen zwischen Substanzabhängigkeit und psychiatrischer Symptomatik und ihrer Bedeutung für Verlauf und Behandlungsergebnis. Konsequenzen für die Versorgung ergeben sich daraus nicht direkt, sondern vermittelt über das 
Verständnis der Komorbidität (Modellvorstellungen). Daher ist die Entwicklung des Verständnisses der Zusammenhänge bzw. Modelle wichtig. Schließlich geht es um die vorgeschlagenen Konsequenzen für Diagnostik, einzelne Behandlungsangebote und das Versorgungssystem.

In der Hamburger Verlaufsuntersuchung zur psychiatrischen Morbidität Opiatabhängiger [8] zeigte sich immerhin bei 55\% eine zusätzliche ICD-10-Diagnose selbst unter Aussparung der Persönlichkeitsstörungen. Ein Drittel der untersuchten Opiatabhängigen zeigte mehrere psychische Störungen und war zusätzlich zur Sucht psychiatrisch behandlungsbedürftig.

Es ist beachtlich, dass im Kontakt zum Behandlungssystem im Verlauf eine Stabilisierung auch der psychisch schwer alterierten Opiatabhängigen erfolgt und, wenn auch mit Abstrichen, eine Besserung im Konsumverhalten sowie in der Entwicklung verschiedener Lebensbereiche $\mathrm{zu}$ verzeichnen ist [9]. Dies mahnt, nicht allein auf die negativen Aspekte zu fokussieren, sondern vor dem Hintergrund bereits diskutierter Hypothesen in Richtung erfolgreicher Selbstheilungsversuche [10] oder Selbstmedikationsprozesse [11] weitere Untersuchungen folgen zu lassen.

\section{Die Etappen der Komorbiditätsforschung}

Das Thema Komorbidität als praktisches und wissenschaftliches Problem der letzten 20 Jahre hat unterschiedliche Wurzeln. Im internationalen Sprachraum rückte die Komorbidität von Substanzabhängigkeit und psychischen Störungen bereits in den 80er Jahren in evaluativen Untersuchungen in den Mittelpunkt $[12,13]$. Die breitere Diskussion begann mit einer Phase der Epidemiologie zur Koinzidenz psychiatrischer Erkrankungen und süchtigen Verhaltens. Im Mittelpunkt standen zunächst die Prävalenzzahlen in verschiedenen Populationen. Es wurde aufgezeigt, dass unter Drogenabhängigen psychische Störungen (nach ICD-10 oder DSM-III-R) weitaus häufiger auftreten als in der so genannten Normalbevölkerung $[14,15,16]$.

Im deutschen Sprachraum erfolgte der Einstieg in die Komorbiditätsforschung zunächst über den erhöhten zusätzlichen Substanzkonsum bei Patienten mit psychiatrischen Störungen [17]. Bei Drogenabhängigen spielte der Zusammenhang von Sucht und psychischen Störungen oder Beeinträchtigungen im Rahmen der zahlreichen Methadon-Evaluationsstudien eine bedeutende Rolle [18-21]. So wurde z.B. unter den Essener Methadonpatienten neben der eigentlichen Suchtdiagnose in über $80 \%$ der Fälle mindestens eine weitere psychiatrische Diagnose gestellt [22]. Im Rahmen der Substitutionsbehandlung haben Diskussionen zum Stellenwert der diagnostizierten psychiatrischen Störungen zu ersten Überlegungen therapeutischer Konsequenzen geführt. So ist die psychotherapeutische (Begleit-)Behandlung [23] oder der Einsatz entsprechender Psychopharmaka im Setting der Substitutionstherapie [24] nicht mehr auf Einzelfälle beschränkt.

Wichtige Ausgangspunkte der Komorbiditätsforschung sind die praktischen therapeutischen (Grenz-)Erfahrungen in verschiedenen Behandlungseinrichtungen und das Bestreben, mögliche Ursachen dieser Schwierigkeiten aufzudecken. Dem folgen (epidemiologische) Untersuchungen zu den Themenbereichen erhöhter Substanzkonsum bei psychiatrischen Pa- tienten einerseits und erhöhte psychiatrische Belastung bei Abhängigen andererseits. Die Komorbiditätsforschung hat die stärkere Hinwendung der Psychiatrie zu Drogenabhängigen seit Ende der 80er Jahre (nach Jahren der Untätigkeit bzw. Hilflosigkeit der Psychiatrie gegenüber diesen Gruppen) zur Voraussetzung. Zunächst wurden die bestehenden Konzeptionen und Klassifikationskonzepte zu Suchterkrankungen (Persönlichkeitsstörungen, neurotische Störungen) auf diese Gruppen bezogen. Mit der Entwicklung zur deskriptiven Psychopathologie und der parallelen Beschreibung verschiedener Syndromgruppen (ICD-10, DSM-III-R), anstatt der Rückführung verschiedener Symptome auf eine Grundstörung, nahm die Komorbiditätsforschung im engeren Sinne ihren Anfang. Bis in die 60er Jahre waren die Süchte Unterformen „soziopathischer Persönlichkeitsstörungen“ (im DSM-I der American Psychiatric Association von 1952 als „soziopathic personality disorders“). Ab 1968 wurde Alkoholismus eine eigenständige Unterform von Persönlichkeitsstörungen; ab 1980 wurden Süchte - unterteilt nach Missbrauchs- und Abhängigkeitskategorien - eigenständig erfasst.

Die grundlegende Hypothese der Forschung der 90er Jahre besteht darin, dass bei Substanzabhängigen psychische Beeinträchtigungen mit erhöhtem Konsum, ungünstigeren Bedingungen bzw. stärkeren Belastungen im sozialen Lebensumfeld und Behandlungsschwierigkeiten einhergehen. Komorbidität in diesem Sinne wurde an verschiedenen (Behandlungs-)Populationen untersucht, und die aus der Wechselwirkung der beiden Syndrome resultierenden Komplikationen für die Behandlung rückten zumindest in der bundesdeutschen Diskussion in den Mittelpunkt. Zahlreiche Studien stellten fest, dass psychische Störungen oder Beeinträchtigungen die Wirkungen drogentherapeutischer Maßnahmen negativ beeinflussen [25-27]. Die Rolle psychiatrischer Komorbidität für Entstehung und Verlauf von Drogenkarrieren konnte auch in bundesdeutschen Studien aufgezeigt werden $[18,21,28]$. Die heute vorherrschende Auffassung zum Wechselverhältnis von Suchtkarriere und psychiatrischer Problematik betont die negativen Aspekte. Der früher erörterte Aspekt (erfolgreicher) Selbstheilungsversuche [10] spielt kaum noch eine Rolle: „With regard to chronic opiate use, the lack of severe long-term psychiatric symptoms in our patients is intriguing (...) However, the opiates may have a medicinal or even prophylactic effect against psychiatric illness." Hier wird die übliche Gerichtetheit von psychischen Beeinträchtigungen und erhöhtem Konsum im Sinne einer Abwärtsspirale hinterfragt.

\section{Zum Verständnis der Bedeutung von Komorbidität oder die Frage der Modellbildung}

Behandlungskonsequenzen ergeben sich aus den festgestellten Zusammenhängen von Komorbidität und Behandlungsproblemen nicht unmittelbar, sondern präzisieren sich erst mit einem (theoretischen) Verständnis von Komorbidität. Was bedeutet psychiatrische Komorbidität? Wie wird der beobachtete Zusammenhang zwischen dem Ausmaß psychischer Störungen und Symptome und dem Drogenkonsum bzw. mit dem Konsum zusammenhängender Probleme in verschiedenen Lebensbereichen verstanden? Bezüglich der Behandlungskonsequenzen geht es um Fragestellungen der Funktion und Bedeutung psychiatrischer Störungen oder psychischer Belastungen in Verbindung mit der Abhängigkeit 
und in unterschiedlichen biografischen und aktuellen Lebenskonstellationen.

In theoretischen Arbeiten der 80er Jahre wird eine unterschiedliche Funktionalität der Droge in Beziehung zur psychischen Situation bzw. zur Psychopathologie angenommen. Es bestehen drei allgemeine Modelle zum Zusammenhang [29-32]:

1. Eine bestehende „primäre“ psychiatrische Erkrankung erhöht generell das Risiko für Missbrauchsverhalten. Die psychopathologisch fassbare Diagnose ist dann nicht nur als ein Risikofaktor für eine „sekundäre“ Abhängigkeitsentwicklung anzusehen, sondern sie beeinflusst auch die Form des Verlaufs, die gesamte Symptomatik und die Prognose der Abhängigkeit negativ [33].

2. Eine „primär“ bestehende Abhängigkeitsproblematik kann zur Entwicklung einer „sekundären“ psychiatrischen Erkrankung beitragen oder diese sogar induzieren. Die diesem Modell zuzuordnenden psychiatrischen Erkrankungen - etwa die so genannten „drogeninduzierten Psychosen“ sind zwar umfangreich untersucht worden [34,35], scheinen aber seltener durch die Drogeneinnahme verursacht als vielmehr (aufgrund vorhandener individueller Dispositionen) durch die Substanzeinnahme ausgelöst oder verstärkt zu sein [36].

3. Als weitere Möglichkeit wird ein gleichzeitiges Nebeneinander von psychiatrischer Erkrankung und stofflichem Missbrauch angenommen, ohne dass eine kausale Beziehung bzw. eine Wechselwirkung zwischen beiden bestehen müsse.

Lehmann et al. [32] ergänzen diese drei Modellvorstellungen durch eine vierte mit der These von einer möglichen gemeinsamen Ätiologie: Danach gebe es Patienten, die aufgrund eines gemeinsamen Faktors eine Prädisposition für die Entwicklung sowohl einer Abhängigkeitserkrankung als auch einer psychiatrischen Erkrankung besitzen. Hier sehen die Autoren die Möglichkeiten eines gemeinsamen genetischen Faktors, einer gemeinsamen biologischen Ätiologie oder psychosozialer Faktoren (wie z.B. Obdachlosigkeit und Armut).

In einzelnen Bereichen psychopathologischer Diagnosen und Drogenkonsum liegen Forschungsergebnisse und Hypothesen zu Zusammenhängen vor - beispielsweise zur Koinzidenz affektiver Störungen und stofflichem Missbrauch [37]. Bei Opiatabhängigen mit der Diagnose einer Major Depression gingen bei etwa 6\% diese Episoden dem Drogenkonsum voraus, bei $94 \%$ aber wurde eine „sekundäre“ Depressivität festgestellt [13]. Diese hohe Koinzidenz erklären einige Autoren aus der Ähnlichkeit in verschiedenen psychopathologischen Aspekten sowie der Periodizität im Verlauf und im Symptombild bei Sucht und manisch-depressiven Erkrankungen. Als mögliche Gründe für Suchtpatienten, depressiv $\mathrm{zu}$ werden oder depressive Symptome zu entwickeln, nennt Nace [31]

- die Ähnlichkeit und Parallelität der Symptomatik gerade während des Entzugs mit organischen Faktoren wie Schlafstörungen, Angstzuständen, Erschöpfung und dysphorischer Stimmungslage,

- die Stigmatisierung von Suchtpatienten und die Erkenntnis eines chronischen Krankheitsprozesses und

- die seiner Meinung nach wichtigste Grundlage für Depressionen in frühen Phasen der Behandlung, das Gefühl der Einsamkeit und Leere, das Suchtmittel als „besten Freund“ zu verlieren oder verloren zu haben, ein organisierendes Lebensprinzip aufgeben zu müssen.

Bei diesen Vorstellungen zur Wechselwirkung von Abhängigkeit und anderer psychiatrischer Krankheit tritt das jeweils andere Syndrom in einem funktionellen und dynamisch oft nachvollziehbaren Kontext auf. So ist der Substanzkonsum nichts, was über den Depressiven oder Ängstlichen hereinbricht, sondern ein möglicherweise ungeeigneter Bewältigungsversuch.

Die Komorbiditätsforschung wirft die Frage auf, in welchem Zusammenhang Substanzabhängigkeit mit weiteren abweichenden bzw. „gestörten“ Wahrnehmungs-, Denk- und Handlungsmustern steht, die unter dem - deskriptiven - Konstrukt psychischer Störungen gefasst werden. Bei der Frage nach auslösenden oder aufrechterhaltenden Bedingungen der Drogenabhängigkeit sowie der Suche nach „geeigneten“ Therapieansätzen stoßen Wissenschaftler und Praktiker immer wieder darauf, dass sich bei vielen Klienten - insbesondere den langjährig Drogenabhängigen - das „Problem“ Drogenabhängigkeit als ein vielfältiges Gemisch unterschiedlicher (lebensbiografischer und aktueller) Problem- und Konfliktkonstellationen darstellt, dem mit eindimensionalen Erklärungs- und Behandlungsmustern nicht beizukommen ist. Hilfreich ist die Ausgangsannahme, dass spezifische Konstellationen von Drogenkonsum und psychischen Störungen oder Belastungen nicht isoliert bestehen, sondern als Bestandteile eines komplexen (immer einheitlichen) Handlungsmusters mit der Entwicklung der allgemeinen Lebenspraxis der Klienten zusammenhängen. Diese Komplexität ist ein Grund dafür, dass die allgemein erwarteten direkten Zusammenhänge zwischen psychischen Störungen und dem Ausmaß des Konsums unter Drogenabhängigen nicht immer deutlich sind [9]. Die simple Formel „mehr Belastungen gleich mehr Konsum“ geht nicht auf. Diese Linearitätsannahme greift schon deshalb zu kurz, weil die Zusammenhänge - z.B. von Ängsten und Konsum - keine direkten, sondern immer über die Lebenspraxis vermittelte sind. In der Regel ist davon auszugehen, dass sich zwischen psychischer Befindlichkeit (inklusive der Probleme und Störungen), dem Konsum psychotroper Substanzen und den allgemeinen, altersabhängigen Lebensanforderungen ein relatives Gleichgewicht einstellt, das in krisenhaften Situationen und Störungen individueller Bewältigungsmuster gefährdet ist. Dies gilt vor allem dann, wenn durch die Häufung von psychosozialen Belastungen, die bei Drogenabhängigen im Vergleich zur Normalpopulation festgestellt werden konnte [38], in Verbindung mit beeinträchtigten individuellen Ressourcen und Mechanismen gesellschaftlicher Ausgrenzung der Wirkung und Funktion des Drogengebrauchs ein größerer Bedeutungswert zukommt.

\section{Die nächste Etappe: Konsequenzen aus Modellprojekten und Komorbiditätsforschung}

Seit den 90er Jahren gibt es einige wichtige klinische Pilotprojekte sowie Veränderungen im Bereich der Allgemeinpsychiatrie in Bezug auf den Umgang mit Patienten mit einer Komorbiditätsproblematik. Auf dem jetzt erreichten Stand der Forschung sowie der Modellprojekte ergeben sich aus unserer Sicht vor allem folgende Aufgaben für die nächste Phase der Entwicklung: 


\section{Verbesserung von Screening und Diagnostik}

Patienten mit einer komorbiden Störung betreffen verschiedene Teile des Hilfesystems. Die Mehrheit kommt zuerst mit Hausärzten in Kontakt sowie dem Suchthilfesystem oder der Notfallmedizin. Da ein rechtzeitiges, spezifisches Therapieangebot von großer Bedeutung für den weiteren Verlauf ist, ist eine der wichtigsten Aufgaben die Entwicklung von in der Praxis verwendbaren und ökonomischen Screening-Instrumenten, die es nicht speziell ausgebildeten Mitarbeiterinnen und Mitarbeitern ermöglichen, die Notwendigkeit weiterführender Diagnostik und fachärztlicher Behandlung zu erkennen. Auch die Operationale Diagnostik psychischer Störungen nach ICD-10 ist insbesondere für den Bereich der Persönlichkeitsstörungen verbesserungswürdig, wenngleich das „robusteste“ Untersuchungsinstrument eine klinische Beurteilung (und Untersuchung) durch entsprechend ausgebildete (Fach-)Ärzte nicht ersetzen kann.

\section{Entwicklung der Epidemiologie}

Die amerikanischen Untersuchungen, insbesondere die „Epidemiological Catchment Area Study“ [14] und der „National Comorbidity Survey“ $[16,39]$ haben gezeigt, wie bedeutsam auch Bevölkerungsuntersuchungen für die Einschätzung der Entwicklung sind. Reine Prävalenzstudien in Behandlungspopulationen sind wenig aussagekräftig. Im Suchtbereich haben die Bemühungen der europäischen Drogenbeobachtungsstelle in Lissabon sowie die Förderung der Epidemiologie im laufenden Suchtforschungsschwerpunkt der Bundesregierung spürbar zur Qualifizierung der Epidemiologie beigetragen. Trotzdem sind regelmäßige und differenzierte Bevölkerungsuntersuchungen sowie Daten über den Verlauf gerade von multimorbiden Störungen außerordentlich wichtig für die weitere Behandlungsplanung. Dazu bedarf es sowohl einer engeren Kooperation zwischen den wissenschaftlichen Zentren in der Bundesrepublik als auch einer deutlichen Verbesserung der Dokumentation in allen Einrichtungen der Suchthilfe. Die regelmäßige Analyse dieser Daten kann auch helfen, Ressourcen sinnvoller einzusetzen und Reformbedarfe im Hilfesystem zu erkennen.

\section{Evaluation von Modellprojekten, Interventionsforschung}

In den letzten Jahren hat es in der Bundesrepublik Deutschland auf Initiative verschiedener Träger neue Behandlungsprojekte gegeben, die sich den spezifischen Problemen verschiedener Gruppen komorbider Patienten zugewendet haben. Die Palette reicht von komplementären Einrichtungen über Akutstationen für psychotische Patienten mit einer Suchtproblematik bis hin zu entsprechenden ambulanten Diensten. Es ist mittlerweile ausreichend Zeit verstrichen, um deren Arbeitsergebnisse zu evaluieren und daraus Schlussfolgerungen für die weitere Entwicklung der Psychiatrie bzw. des Hilfesystems insgesamt zu erarbeiten. Darüber hinaus wäre es wichtig, den Entwicklungsprozess in der allgemeinen Psychiatrie, der Notfallmedizin sowie der ambulanten Suchthilfe daraufhin zu untersuchen, auf welche Weise der Umgang mit komorbiden Patienten erfolgt und welche Bedürfnisse es für die Weiterentwicklung des Versorgungssystems gibt. Dazu wären auch Kosteneffektivitätsuntersuchungen z.B. im Rahmen der vom BMBF geplanten Suchtforschungsnetze - ein wichtiger Beitrag.
Spezielle Modelleinrichtungen und -projekte sind derzeit nicht in ausreichender Anzahl vorhanden. Dies betrifft die Psychopharmakotherapie, das Behandlungssetting sowie Psycho- und Soziotherapie. Bei der Relevanz der Komorbiditätsproblematik ist dies ein Problem sowohl der Allgemeinpsychiatrie als auch des allgemeinen Versorgungssystems, und es ist notwendig, diese Gesamtstrukturen weiterzuentwickeln, wie dies auch in anderen Ländern erfolgt. (Die in den USA entwickelte „Dialektische Verhaltenstherapie“ [nach Linnehan] hat z.B. im Rahmen eines Amsterdamer Therapieprojekts außerordentlich interessante Ergebnisse bei der ambulanten Behandlung von Suchtpatienten mit einer Borderline-Störung erbracht.) Auf dieser Grundlage wäre es sinnvoll, in den nächsten Jahren eine verstärkte interdisziplinäre Diskussion über Behandlungsstandards in den verschiedenen Bereichen zu führen.

\section{4. Ätiologische Modelle und interdisziplinäre Modellbildung}

Die mehr klinisch orientierten Forschungsansätze sind ein notwendiger erster Schritt. Sie sollten darüber hinaus auch zu einer interdisziplinären Diskussion über Einflussfaktoren und Erfolgskriterien von Therapieverläufen führen, die die Weiterentwicklung eines qualifizierten und differenzierten Therapieangebots zum Ziel haben. Es ist bemerkenswert, dass gerade das Verhältnis von psychischen Störungen und Sucht viele Autoren zu Überlegungen übergreifender Modellbildungen über psychische Störungen anregt. Im Austausch mit der klinischen Psychologie, den Sozialwissenschaften sowie der Grundlagenforschung lassen sich in diesem Punkt interessante Entwicklungen für die nächsten Jahre erwarten.

Die neue Zeitschrift „Suchttherapie“ kann hier einen wesentlichen Beitrag zur Verbindung von Forschung und Interventionen sowie von Theoriebildung und Optimierung der Versorgung leisten.

\section{Literatur}

${ }^{1}$ Woody GE, McLellan AT, OBrien CP. Treatment of behavioral and psychiatric problems associated with opiate dependence. NIDA Research Monograph Series, 1984: 23-35

2 Alterman AI (Hrsg). Substance abuse and psychopathology. New York: Plenum Press, 1985

${ }^{3}$ Krausz M, Müller-Thomsen T. Komorbidität - Therapie von psychischen Störungen und Sucht. Konzepte für Diagnostik, Behandlung und Rehabilitation. Freiburg: Lambertus, 1994

${ }^{4}$ Dilling $\mathrm{H}$, Mombour W, Schmidt MH (Hrsg). Weltgesundheitsorganisation. Internationale Klassifikation psychischer Störungen. ICD-10 Kapitel V (F). Klinisch-diagnostische Leitlinien. 3. Aufl. Bern: Hans Huber, 1999

5 Verheul R, van den Brink W, Hartgers C. Prevalence of personality disorders among alcoholics and drug addicts: an overview. European Addiction Research 1995; 1: 166-177

${ }^{6}$ Fiedler P. Persönlichkeitsstörungen. Weinheim: Beltz, 1994

7 Verheul R. The Role of Diagnosing Personality Disorders in Substance Abuse Treatment. Prevalence, Diagnostic Validity and Clinical Implications. Amsterdam: Thesis Publishers, 1997

${ }^{8}$ Krausz M, Verthein U, Degkwitz P. Prävalenz psychischer Störungen bei Opiatabhängigen mit Kontakt zum Drogenhilfesystem. Nervenarzt 1998; 69: 557-567

9 Verthein U, Degkwitz P, Krausz M. Psychische Störungen und Verlauf der Opiatabhängigkeit. Psychiatrische Praxis 2000; 27 $77-85$ 
${ }^{10}$ McLellan AT, Childress AR et al. Drug Abuse and Psychiatric Disorders. In: Alterman AI (Hrsg). Substance abuse and psychopathology, New York: Plenum Press, 1985: 137-172

${ }^{11}$ Khantzian EJ. The self-medication hypothesis of addictive disorders: focus on heroin and cocaine dependence. American Journal of Psychiatry 1985; 142: 1259-1264

12 McLellan AT, Woody GE, O'Brien CP. Development of psychiatric illness in drug abusers. New England Journal of Medicine 1979; 201: $1310-1314$

13 Rounsaville BJ, Weissman MM, Kleber H, Wilber C. Heterogenity of Psychiatric Diagnosis in Treated Opiate Addicts. Archives of General Psychiatry 1982; 39: 161-166

${ }^{14}$ Regier DA, Farmer ME, Rae DS, Locke BZ, Keith SJ, Judd LL, Goodwin FK. Comorbidity of mental disorders with alcohol and other drug abuse. Results from the Epidemiologic Catchment Area (ECA) Study. Journal of the American Medical Association 1990; 264: 2511-2518

${ }^{15}$ Wittchen H-U, Essau CA, von Zerssen D, Krieg CJ, Zaudig M. Lifetime and six-months prevalence of mental disorders in the Munich Follow-up Study. European Archives of Psychiatry and Clinical Neuroscience 1992; 241: 247-258

${ }^{16}$ Warner LA, Kessler RC, Hughes M, Anthony JC, Nelson CB. Prevalence and correlates of drug use and dependence in the United States. Results from the National Comorbidity Survey. Archive of General Psychiatry 1995; 52: 219-229

17 Schwoon DR, Krausz M. Suchtkranke - Die ungeliebten Kinder der Psychiatrie. Stuttgart: Enke, 1990

18 Ministerium für Arbeit, Gesundheit und Soziales des Landes Nordrhein-Westfalen (Hrsg). Wissenschaftliches Erprobungsvorhaben medikamentengestützte Rehabilitation bei i.v. Opiatabhängigen. Köln: Abschlussbericht, 1993

19 Zenker C, Lang P. Methadon-Substitution in Bremen. Abschlussbericht der sozialmedizinischen Begleitforschung. Bremen: Bremer Institut für Präventionsforschung und Sozialmedizin BIPS, 1995

${ }^{20}$ Raschke P. Substitutionstherapie - Ergebnisse langfristiger Behandlung von Opiatabhängigen Freiburg. Lambertus, 1994

${ }^{21}$ Raschke P, Chorzelski G, Brinkmann R. Beikonsum, Selbstmedikation und Medikation. In: Gölz J (Hrsg). Moderne Suchtmedizin, Stuttgart: Thieme: 1998: C3.8.2.-1-C3.8.2.-13

22 Rösinger C, Gastpar M. Methadon-Substitution in der Behandlung schwerkranker Opiatabhängiger. Deutsches Ärzteblatt 1991; 88: 2462-2469

${ }^{23}$ Scherbaum N, Bender S. Der Stellenwert der Psychotherapie im Rahmen der Substitutionsbehandlung mit Methadon. Sucht 1995; 41: 18-24

24 Gölz J. Der drogenabhängige Patient. 2. Aufl. München: Urban \& Fischer, 1999

${ }^{25}$ McLellan AT, Luborsky L, Woody GE, O’Brien CP, Druley KA. Predicting response to alcohol and drug abuse treatments. Role of psychiatric severity. Archives of General Psychiatry 1983; 4: 620-625

${ }^{26}$ Rounsaville BJ, Kosten TR, Weissman MM, Kleber HD. Prognostic significance of psychopathology in treated opiate addicts. Archives of General Psychiatry 1986; 43: 739-745

27 Darke S, Swift W, Hall W. Prevalence, severity and correlates of psychological morbidity among methadone maintenance clients. Addiction 1994; 89: 211-217

${ }^{28}$ Verthein U, Degkwitz P, Kühne A, Krausz M. Komorbidität von Opiatabhängigkeit und psychischen Störungen - Ergebnisse einer Verlaufsuntersuchung. Sucht 1998; 44: 232-246

${ }^{29}$ Freed EX. Alcoholism and schizophrenia: the search for perspectives. Journal of Studies on Alcohol 1975; 36: 853-881

30 Schuckit MA. Alcoholism and other psychiatric disorders. Hospital and Community Psychiatry 1983; 34: 1022-1027

${ }^{31}$ Nace EP. The treatment of alcoholism. New York: Brunner/Mazel, 1987
${ }^{32}$ Lehman AF, Myers CP, Corty E. Assessment and classification of patients with psychiatric substance abuse syndromes. Hospital and Community Psychiatry 1989; 4: 1019-1025

${ }^{33}$ Lieberman JA, Bowers MB. Substance abuse among schizophrenia: editors introduction. Schizophrenia Bulletin 1990; 16: 29-30

${ }^{34}$ Bron B, Fröscher W, Gehlen W. Differentialdiagnostische und syndromgenetische Probleme and Aspekte drogenindizierter Psychosen bei Jugendlichen. Fortschr Neurol Psychiat 1976; 12: 673-682

35 Täschner KL. Zur Psychopathologie und Differentialdiagnose so genannter Cannabispsychosen. Fortschr Neurol Psychiat 1983; 51: 248-253

${ }^{36}$ Kleiber D, Kovar KA. Auswirkungen des Cannabiskonsums. Eine Expertise zu pharmakologischen und psychosozialen Konsequenzen. Stuttgart: Wissenschaftliche Verlagsgesellschaft, 1998

37 Allen MH, Frances RJ. Varieties of psychopathology found in patients with addictive disorder: a review. In: Meyer RE (Hrsg). Psychopathology and addictive disorders, New York: Guilford, 1986

38 Krausz M, Degkwitz P, Verthein U. Lebensereignisse und psychosoziale Belastungen bis zur Pubertät - Entwicklungsbedingungen Opiatabhängiger und ihrer „normalen“ Altersgenossen. Kindheit und Entwicklung 1998; 7: 221-230

${ }^{39}$ Kessler RC, McGonagle KA, Zhao S, Nelson CB, Hughes M, Eshleman S, Wittchen HU, Kendler KS. Lifetime and 12-month prevalence of DSM-III-R psychiatric disorders in the United States. Results from the National Comorbidity Survey. Arch Gen Psychiatry $1994 ; 51: 8-19$

\section{Prof. Dr. Michael Krausz}

Zentrum für Interdisziplinäre Suchtforschung der Universität Hamburg (ZIS) c/o Zentrum für Psychosoziale Medizin, Psychiatrie und Psychotherapie im UKE Martinistraße 52 20246 Hamburg E-mail: krausz@uke.uni-hamburg.de 